\title{
Determining the Scope of Recovery from Physically Damaged Micro SD Card
}

\author{
Amar B. Landepatil, Shobha K. Bawiskar
}

\begin{abstract}
Today's use of secondary devices like cloud storage, Hard Disk, Pen Drive, SD, CDs, DVDs is constantly growing. Data might be deleted, loss or device is formatted accidentally or intentionally by various means. It's a myth that "once secondary storage devices are damaged then there is no access to data too". This article gives the detail investigation that from damaged devices too data can be recovered.
\end{abstract}

Keywords: Cyber crime, Data Loss, MICRO SD CARD, Recovery software's.

\section{INTRODUCTION}

Cyber Crime is technology based crime committed by technocrats.[1] The electronic crime scene that possess digital and electronic devices creates new challenges for the investigator [2].Dependency of data on storage devices is at hike. As per the studies Flash memory market revenues worldwide from 2013 to 2021 (in billion U.S. dollars) Release date October 2017 [3] as shown in Fig 1.

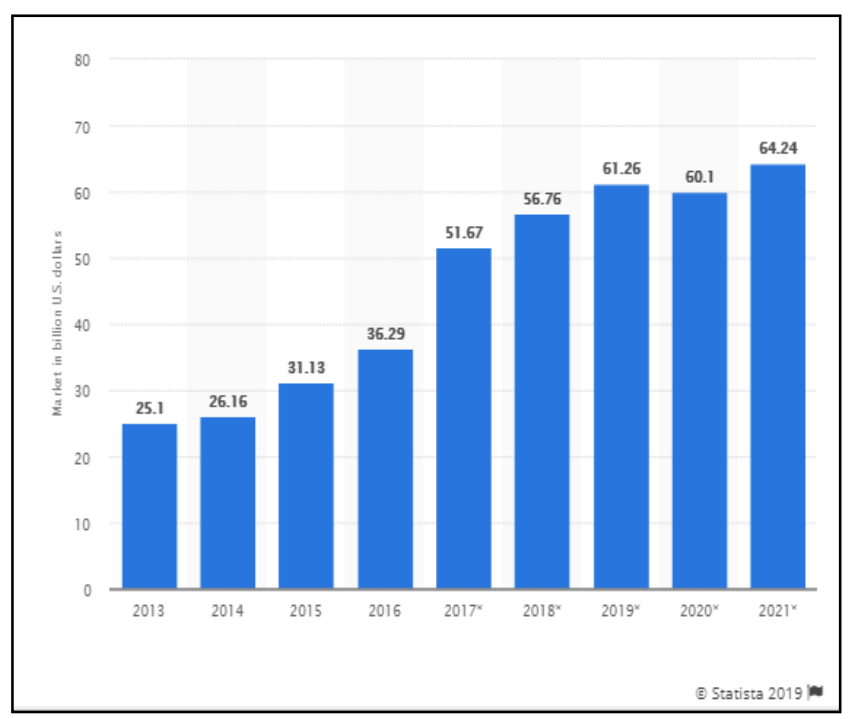

Fig 1- Flash memory market size worldwide 2013-2021

Criminals are doing smart crimes via cyber as a media and/or as a tool, and after committing crimes the criminal mindset is to destroying the digital evidence. Any data once saved in e-storage device is always recoverable in case if data is loosed.

Data loss is an error condition in information systems in which information is destroyed by failures or neglect in storage, transmission, or processing.

Revised Manuscript Received on February 05, 2020.

* Correspondence Author

Amar B. Landepatil*, Department of Digital and Cyber Forensic, Government Institute of Forensic Science, Aurangabad (MS). India.

E-mail: Amarnathlandepatil43@gmail.com

Dr. Shobha K. Bawiskar, Department of Digital and Cyber Forensic, Government Institute of Forensic Science, Aurangabad (MS). India.

E-mail: Shobha bawiskar@yahoo.co.in.

(c) The Authors. Published by Blue Eyes Intelligence Engineering and Sciences Publication (BEIESP). This is an open access article under the CC BY-NC-ND license (http://creativecommons.org/licenses/by-nc-nd/4.0/)

Information systems implement backup and disaster recovery equipment and processes to prevent data loss or restore lost data.[4]

Most commonly data loss causes due to following reasons:

- Accidental deletion of files or folders

- $\quad$ File system formatting

- Logical damage of a file system

- Loss of information about partition

- Storage failure

- $\quad$ physical damage to devices [5]

This article will be focused on physical damages to storage device like memory card 16 GB and looking for the chances to recover the data $99.99 \%$. as one percent chances are there to get negative results in exceptional cases. Data Recovery refers to a situation of recovering specific information which becomes inaccessible due to logical or physical damages of the targeted storage device.[6,8]

\section{MICRO SD CARD}

The Secure Digital Card is a flash-based memory card that is specifically designed to meet the security, capacity, performance and environmental requirements inherent in newly emerging audio and video consumer electronic devices. The SD Card includes a copyright protection mechanism that complies with the security of the SDMI standard, and is faster and capable of higher Memory capacity. SD Card Capacities $16 \mathrm{MB}$, $32 \mathrm{MB}, 64 \mathrm{MB}$, $128 \mathrm{MB}$, $256 \mathrm{MB}, 512 \mathrm{MB}, 1024 \mathrm{MB}$ etc[7]

\section{EXPERIMENTAL SETUP}

Aims: To Recover (deleted or formatted) data from MICRO SD CARD.

Background: In this research article secondary storage device like MICRO SD CARD of $16 \mathrm{~GB}$ is used for experimental work. 25 MICRO SD CARDs are used first it was formatted then on each one GB data inserted which includes all types of multimedia files. Then physically MICRO SD CARDs was damaged via various methods like heating, freezing ...etc.

Data Sample: Multimedia content of one GB (1-GB data) with Maximum types of file extensions are collected as data sample.

Secondary Device used: 25 SanDisk's Cruzer Blade ${ }^{\mathrm{TM}}$ USB Flash Drive of 16 GB (MICRO SD CARD).

Hypothesis: Data cannot be recovered from physically damaged MICRO SD CARD memory card 
2. Procedure applied on Samples collected to physically

damage the samples: details shown in Table 2.1 to 2.5

2.1 Method 1: Buried in Mud Method - in this devices were

buried in mud with varying conditions as shown in Table 2.1

Table 2.1 Buried in Mud Method

\begin{tabular}{|c|c|c|c|c|}
\hline Sample No & Mud & Time Factor & Detectable & Recovery possibility \\
\hline Sample 1 & dry & 1hr & Yes & Yes \\
\hline Sample 2 & dry & 1-day= 24hrs & Yes & Yes \\
\hline Sample 3 & Semi wet & 1hr & Yes & Yes \\
\hline Sample 4 & Semi Wet & 5hrs & Yes & Yes \\
\hline Sample 5 & Liquid muddy & 7-days=168 hrs & Yes & \multicolumn{2}{|c|}{} \\
\hline
\end{tabular}

2.2 Method 2: Use of aqueous media like Water, in this devices were deep in water varying conditions as shown in Table 2.2

Table 2.2 Use of aqueous media

\begin{tabular}{|l|l|l|l|l|l|l|}
\hline $\begin{array}{l}\text { Sample } \\
\text { No }\end{array}$ & Water amount & $\begin{array}{l}\text { Water } \\
\text { Type }\end{array}$ & $\begin{array}{l}\text { Environment } \\
\text { temperature } \\
\text { approximately }\end{array}$ & $\begin{array}{l}\text { Time depth } \\
\text { Factor }\end{array}$ & Detected & $\begin{array}{l}\text { Recovery } \\
\text { possibility }\end{array}$ \\
\hline Sample 1 & 4 ltr in bucket & Normal Water & $6-8$ & $1 \mathrm{hr}$ & yes & yes \\
\hline Sample 2 & 4 ltr in bucket & Normal Water & $8-11$ & $12 \mathrm{hrs}$ & yes & yes \\
\hline Sample 3 & $500 \mathrm{ml}$ in bottle & Drainage Water & $8-14$ & $12 \mathrm{hrs}$ & yes & yes \\
\hline Sample 4 & $\begin{array}{l}500 ~ \mathrm{ml} \mathrm{in} \\
\text { beaker }\end{array}$ & $\begin{array}{l}\text { Hot } 50 \text { continuously } \\
\text { boiled Water }\end{array}$ & $11-14$ & $1 \mathrm{hr}$ & yes & yes \\
\hline Sample 5 & $\begin{array}{l}500 ~ \mathrm{ml} \text { in } \\
\text { beaker }\end{array}$ & $\begin{array}{l}\text { Hot 100 continuously } \\
\text { boiled Water }\end{array}$ & $11-14$ & $1 \mathrm{hr}$ & No & No \\
\hline
\end{tabular}

2.3 Method 3: Heating Method-, in this devices were kept in hot oven with various temperature as well as burned used Bunsen burner varying conditions as shown in Table 2.3

Table 2.3 Heating Method

\begin{tabular}{|l|l|l|l|l|l|}
\hline $\begin{array}{l}\text { Sam } \\
\text { ple } \\
\text { No }\end{array}$ & $\begin{array}{l}\text { Heating } \\
\text { equipm } \\
\text { ent } \\
\text { used }\end{array}$ & $\begin{array}{l}\text { Temper } \\
\text { ature in } \\
\text { Celsius }\end{array}$ & $\begin{array}{l}\text { Tim } \\
\text { e } \\
\text { Fact } \\
\text { or }\end{array}$ & $\begin{array}{l}\text { Detect } \\
\text { able }\end{array}$ & $\begin{array}{l}\text { Reco } \\
\text { very } \\
\text { possi } \\
\text { bility }\end{array}$ \\
\hline $\begin{array}{l}\text { Sam } \\
\text { ple 1 }\end{array}$ & $\begin{array}{l}\text { Hot } \\
\text { Oven } \\
\text { Method }\end{array}$ & 50 & $1 \mathrm{hr}$ & yes & Yes \\
\hline $\begin{array}{l}\text { Sam } \\
\text { ple 2 }\end{array}$ & $\begin{array}{l}\text { Hot } \\
\text { Oven } \\
\text { Method }\end{array}$ & 80 & $1 \mathrm{hr}$ & yes & Yes \\
\hline $\begin{array}{l}\text { Sam } \\
\text { ple 3 }\end{array}$ & $\begin{array}{l}\text { Hot } \\
\text { Oven } \\
\text { Method }\end{array}$ & 160 & $1 \mathrm{hr}$ & yes & Yes \\
\hline $\begin{array}{l}\text { Sam } \\
\text { ple 4 }\end{array}$ & $\begin{array}{l}\text { Bunsen } \\
\text { Burner }\end{array}$ & heated & $\begin{array}{l}05 \\
\text { sec }\end{array}$ & yes & Yes \\
\hline $\begin{array}{l}\text { Sam } \\
\text { ple 5 }\end{array}$ & $\begin{array}{l}\text { Bunsen } \\
\text { Burner }\end{array}$ & heated & $\begin{array}{l}60 \\
\text { min }\end{array}$ & Yes/no & No \\
\hline
\end{tabular}

2.4 Method 4: Freezing Method-, in this devices were freezed varying conditions as shown in Table 2.4

Table 2.4 Freezing Method

\begin{tabular}{|l|l|l|l|l|}
\hline ample No & $\begin{array}{l}\text { Freezer } \\
\text { Temper } \\
\text { ature in } \\
\text { degree } \\
\text { Celsius }\end{array}$ & $\begin{array}{l}\text { Time } \\
\text { Fact } \\
\text { or }\end{array}$ & $\begin{array}{l}\text { Detectabl } \\
\text { e }\end{array}$ & $\begin{array}{l}\text { Recover } \\
\text { y } \\
\text { possibili } \\
\text { ty }\end{array}$ \\
\hline Sample 1 & -20 & 1 & Yes & Yes \\
\hline Sample 2 & -20 & 24 & Yes & Yes \\
\hline Sample 3 & -20 & 48 & Yes & Yes \\
\hline Sample 4 & -20 & 72 & Yes & Yes \\
\hline
\end{tabular}

\begin{tabular}{|l|l|l|l|l|}
\hline Sample 5 & -20 & 96 & Yes & Yes \\
\hline
\end{tabular}

2.5 Method 5: Scratching Method, in this devices were scratched with i-Pin for number of times as shown in Table 2.5

Table 2.5 Scratching Method

\begin{tabular}{|l|l|l|l|}
\hline $\begin{array}{l}\text { Sample } \\
\text { No }\end{array}$ & $\begin{array}{l}\text { No of } \\
\text { Scratches } \\
\text { with I-pin }\end{array}$ & $\begin{array}{l}\text { Detecta } \\
\text { ble }\end{array}$ & $\begin{array}{l}\text { Recovery } \\
\text { possibilit } \\
\text { y }\end{array}$ \\
\hline Sample 1 & 100 & Yes & Yes \\
\hline Sample 2 & 150 & Yes & Yes \\
\hline Sample 3 & 200 & Yes & Yes \\
\hline Sample 4 & 250 & Yes & Yes \\
\hline Sample 5 & 500 & No & No \\
\hline
\end{tabular}

After that MICRO SD CARDs were checked for its detection, once it is detected three freeware software's and one licesenced software was used

\section{The procedure to recovery data is as follows:}

Step no.1 Select the appropriate software

1. Software Tool No1 - Recuva Freeware[9],

2. Software Tool No 2 - Photorec 7.0 Data Recovery Freeware[11],

3. Software Tool No 3 - 7-Data Recovery[10],

4. Software Tool No 4 - Stellar Phoenix Licensed[12]

Step no.2 Select the secondary device like MICRO SD CARD

Step no.3 If device are detected goto step no 4 else goto to step no. 5 
Step no 4

Step no.4.1 Scan the device

Step no.4.2 Content will be shown in either unsuccessful recovery, partially successful recovery or completely successful recovery
Step no.4.4 Select the location where the recovered data have to be saves

Step no. 4.5 End

Step no.5 Try for device detection if detected goto to step no 4 else goto step no 6

Step no.6 End

Step no.4.3 Select specific files or devices that want to be recovered.

Observation: - shown in Table 3.1 to 3.5

Table 3.1 Buried in Mud Method

Method 3.1: Buried in Mud Methods

\begin{tabular}{|c|c|c|c|c|c|c|c|c|c|c|c|c|c|}
\hline \multirow[t]{2}{*}{$\begin{array}{l}\text { Sampl } \\
\text { e No }\end{array}$} & \multirow[t]{2}{*}{ Condition } & \multicolumn{3}{|c|}{$\begin{array}{c}\text { Software Tool No1 } \\
\text { Recuva } \\
\text { Freeware }\end{array}$} & \multicolumn{3}{|c|}{$\begin{array}{c}\text { Software Tool No } 2 \\
\text { Photorec 7.0 Data } \\
\text { Recovery } \\
\text { Freeware } \\
\end{array}$} & \multicolumn{3}{|c|}{$\begin{array}{c}\text { Software Tool No } 3 \\
\text { 7-Data Recovery }\end{array}$} & \multicolumn{3}{|c|}{$\begin{array}{c}\text { Software Tool No } \\
4 \\
\text { Stellar Phoenix } \\
\text { Licensed } \\
\end{array}$} \\
\hline & & DST & $\begin{array}{l}\text { FSE } \\
(269)\end{array}$ & $\mathbf{R}$ & ST & $\begin{array}{c}\text { FSE } \\
(269 \\
)\end{array}$ & $\mathbf{R}$ & ST & $\begin{array}{l}\text { FSE } \\
(269 \\
)\end{array}$ & $\mathbf{R}$ & $\begin{array}{c}\text { DS } \\
\text { T }\end{array}$ & $\begin{array}{c}\text { FS } \\
\text { E }\end{array}$ & $\mathbf{R}$ \\
\hline $\begin{array}{l}\text { Sampl } \\
\text { e } 1\end{array}$ & $\begin{array}{l}\text { Buried in } \\
\text { dry Mud } \\
\text { for 1-hr }\end{array}$ & $\begin{array}{l}3.56 \\
\text { HRS }\end{array}$ & 41 & $\begin{array}{l}9.67 \mathrm{M} \\
\text { B }\end{array}$ & $\begin{array}{l}1.56 \\
\text { HRS }\end{array}$ & 274 & $\begin{array}{l}\text { 989M } \\
\text { B }\end{array}$ & $\begin{array}{l}2.10 \\
\text { HRS }\end{array}$ & 506 & $\begin{array}{l}1.66 \mathrm{G} \\
\mathrm{B}\end{array}$ & $\begin{array}{l}2.1 \\
6 \\
\text { HR } \\
S\end{array}$ & 270 & $\begin{array}{l}\text { 1.0G } \\
B\end{array}$ \\
\hline $\begin{array}{l}\text { Sampl } \\
\text { e } 2\end{array}$ & $\begin{array}{l}\text { Buried in } \\
\text { dry Mud } \\
\text { for } 1- \\
\text { day=24 } \\
\text { hrs }\end{array}$ & $\begin{array}{l}3.45 \\
\text { HRS }\end{array}$ & & $\begin{array}{l}14.5 \mathrm{G} \\
\mathrm{B}\end{array}$ & $\begin{array}{l}1.50 \\
\text { HRS }\end{array}$ & 274 & $\begin{array}{l}\text { 989M } \\
\text { B }\end{array}$ & $\begin{array}{l}1.56 \mathrm{HR} \\
\mathrm{S}\end{array}$ & 506 & $\begin{array}{l}1.66 \mathrm{G} \\
\mathrm{B}\end{array}$ & $\begin{array}{l}2.1 \\
5 \\
\mathrm{HR} \\
\mathrm{S}\end{array}$ & 270 & $\begin{array}{l}\text { 1.0G } \\
B\end{array}$ \\
\hline $\begin{array}{l}\text { Sampl } \\
\text { e } 3\end{array}$ & $\begin{array}{l}\text { Buried in } \\
\text { Semi wet } \\
\text { Mud for } \\
\text { 1-hr }\end{array}$ & $\begin{array}{l}2.13 \\
\text { HRS }\end{array}$ & 295 & $\begin{array}{l}30.6 \mathrm{G} \\
\mathrm{B}\end{array}$ & $\begin{array}{l}1.57 \\
\text { HRS }\end{array}$ & 274 & $\begin{array}{l}989 \mathrm{M} \\
\mathrm{B}\end{array}$ & $\begin{array}{l}2.19 \\
\text { HRS }\end{array}$ & 506 & $\begin{array}{l}1.66 \mathrm{G} \\
\mathrm{B}\end{array}$ & $\begin{array}{l}2.3 \\
0 \\
\text { HR } \\
\text { S }\end{array}$ & 270 & $\begin{array}{l}1.0 \mathrm{G} \\
\mathrm{B}\end{array}$ \\
\hline $\begin{array}{l}\text { Sampl } \\
\text { e } 4\end{array}$ & $\begin{array}{l}\text { Buried in } \\
\text { Semi wet } \\
\text { Mud for } \\
5 \text {-hr }\end{array}$ & $\begin{array}{l}1.13 \\
\text { HRS }\end{array}$ & 9 & $6.4 \mathrm{~GB}$ & $\begin{array}{l}2.14 \mathrm{HR} \\
\mathrm{S}\end{array}$ & 264 & $\begin{array}{l}\text { 989M } \\
\text { B }\end{array}$ & 2.HRS & 506 & $\begin{array}{l}1.66 \mathrm{G} \\
\mathrm{B}\end{array}$ & $\begin{array}{l}1.5 \\
8 \\
\text { HR } \\
\text { S }\end{array}$ & 272 & $\begin{array}{l}1.0 \mathrm{G} \\
\mathrm{B}\end{array}$ \\
\hline $\begin{array}{l}\text { Sampl } \\
\text { e } 5\end{array}$ & $\begin{array}{l}\text { Buried in } \\
\text { Liquid } \\
\text { muddy for } \\
7 \text { - } \\
\text { days=168 } \\
\text { hrs }\end{array}$ & $\begin{array}{l}1.45 \\
\text { HRS }\end{array}$ & 18 & $\begin{array}{l}4.67 \mathrm{G} \\
\mathrm{B}\end{array}$ & $\begin{array}{l}1.45 \\
\text { HRS }\end{array}$ & 80 & $\begin{array}{l}\text { 989M } \\
\text { B }\end{array}$ & $\begin{array}{l}2.40 \\
\text { HRS }\end{array}$ & 506 & $\begin{array}{l}1.66 \mathrm{G} \\
\mathrm{B}\end{array}$ & $\begin{array}{l}2.1 \\
0 \\
\mathrm{HR} \\
\mathrm{S}\end{array}$ & 270 & $\begin{array}{l}1.0 \mathrm{G} \\
B\end{array}$ \\
\hline
\end{tabular}

Deep Scan Time=DST, Total No of Files extracted out of $1 \mathrm{~GB}=\mathrm{FS}$, Recovery status $=\mathrm{R}$

Table 3.2 Use of aqueous media

Method 3.2: Use of aqueous medium like Water $\left(\mathrm{H}_{2} \mathrm{O}\right)$

\begin{tabular}{|c|c|c|c|c|c|c|c|c|c|c|c|c|c|}
\hline \multirow[t]{2}{*}{$\begin{array}{l}\text { Sampl } \\
\text { e No }\end{array}$} & \multirow{2}{*}{$\begin{array}{c}\text { Conditio } \\
\mathbf{n}\end{array}$} & \multicolumn{3}{|c|}{$\begin{array}{c}\text { Software Tool No1 } \\
\text { Recuva } \\
\text { Freeware }\end{array}$} & \multicolumn{3}{|c|}{$\begin{array}{c}\text { Software Tool No } 2 \\
\text { Photorec 7.0 Data } \\
\text { Recovery } \\
\text { Freeware }\end{array}$} & \multicolumn{3}{|c|}{$\begin{array}{l}\text { Software Tool No } 3 \\
\text { 7-Data Recovery }\end{array}$} & \multicolumn{3}{|c|}{$\begin{array}{c}\text { Software Tool No } 4 \\
\text { Stellar Phoenix } \\
\text { Licensed }\end{array}$} \\
\hline & & DST & $\begin{array}{l}\text { FSE } \\
(269 \\
)\end{array}$ & $\mathbf{R}$ & $\begin{array}{c}\text { DS } \\
\mathbf{T}\end{array}$ & $\begin{array}{c}\text { FSE } \\
(269 \\
)\end{array}$ & $\mathbf{R}$ & DST & $\begin{array}{c}\text { FSE } \\
(269 \\
)\end{array}$ & $\mathbf{R}$ & $\begin{array}{c}\mathbf{D S} \\
\mathbf{T}\end{array}$ & $\begin{array}{c}\text { FSE } \\
(269 \\
)\end{array}$ & $\mathbf{R}$ \\
\hline $\begin{array}{l}\text { Sampl } \\
\text { e } 1\end{array}$ & $\begin{array}{l}4 \text { ltr in } \\
\text { bucket }\end{array}$ & $\begin{array}{l}3.45 \\
\text { HRS }\end{array}$ & 264 & $\begin{array}{l}50.5 \mathrm{G} \\
\text { B }\end{array}$ & $\begin{array}{l}1.67 \\
\text { HR } \\
\text { S }\end{array}$ & 263 & $\begin{array}{l}957 \mathrm{M} \\
\mathrm{B}\end{array}$ & $\begin{array}{l}2.15 \\
\text { HRS }\end{array}$ & 285 & $\begin{array}{l}704 \mathrm{M} \\
\text { B }\end{array}$ & $\begin{array}{l}2.58 \\
\text { HR } \\
\text { S }\end{array}$ & 270 & $\begin{array}{l}1.0 \mathrm{G} \\
\mathrm{B}\end{array}$ \\
\hline
\end{tabular}


Determining the Scope of Recovery from Physically Damaged Micro SD Card

\begin{tabular}{|c|c|c|c|c|c|c|c|c|c|c|c|c|c|}
\hline $\begin{array}{l}\text { Sampl } \\
\text { e } 2\end{array}$ & $\begin{array}{l}4 \text { ltr in } \\
\text { bucket }\end{array}$ & $\begin{array}{l}\text { 3.25HR } \\
\mathrm{S}\end{array}$ & 264 & $\begin{array}{l}50.5 \mathrm{G} \\
\mathrm{B}\end{array}$ & $\begin{array}{l}1.30 \\
\mathrm{HR} \\
\mathrm{S}\end{array}$ & 257 & $\begin{array}{l}950 \mathrm{M} \\
\text { B }\end{array}$ & $\begin{array}{l}1.56 \\
\text { HRS }\end{array}$ & 270 & $\begin{array}{l}690 \mathrm{M} \\
\text { B }\end{array}$ & $\begin{array}{l}1.45 \\
\text { HR } \\
\mathrm{S}\end{array}$ & 270 & $\begin{array}{l}1.0 \mathrm{G} \\
\mathrm{B}\end{array}$ \\
\hline $\begin{array}{l}\text { Sampl } \\
\text { e } 3\end{array}$ & $\begin{array}{l}500 \mathrm{ml} \text { in } \\
\text { bottle }\end{array}$ & $\begin{array}{l}3.15 \mathrm{HR} \\
\mathrm{S}\end{array}$ & 48 & $\begin{array}{l}20.8 \mathrm{G} \\
\mathrm{B}\end{array}$ & $\begin{array}{l}1.55 \\
\mathrm{HR} \\
\mathrm{S}\end{array}$ & 258 & $\begin{array}{l}953 \mathrm{M} \\
\mathrm{B}\end{array}$ & $\begin{array}{l}2.16 \mathrm{HR} \\
\mathrm{S}\end{array}$ & 500 & $\begin{array}{l}1.66 \mathrm{G} \\
\text { B }\end{array}$ & $\begin{array}{l}3.15 \\
\text { HR } \\
S\end{array}$ & 270 & $\begin{array}{l}1.0 \mathrm{G} \\
\mathrm{B}\end{array}$ \\
\hline $\begin{array}{l}\text { Sampl } \\
\text { e } 4\end{array}$ & $\begin{array}{l}500 \mathrm{ml} \text { in } \\
\text { beaker }\end{array}$ & $\begin{array}{l}3.5 \\
\text { HRS }\end{array}$ & 144 & $\begin{array}{l}19.8 G \\
B\end{array}$ & $\begin{array}{l}1.57 \\
\mathrm{HR} \\
\mathrm{S}\end{array}$ & 274 & $\begin{array}{l}986 \mathrm{M} \\
\text { B }\end{array}$ & $\begin{array}{l}2.45 \\
\text { HRS }\end{array}$ & 506 & $\begin{array}{l}1.66 \mathrm{G} \\
\text { B }\end{array}$ & $\begin{array}{l}2.45 \\
\text { HR } \\
S\end{array}$ & 270 & 1.0GB \\
\hline $\begin{array}{l}\text { Sampl } \\
\text { e } 5 \\
\end{array}$ & $\begin{array}{l}500 \mathrm{ml} \text { in } \\
\text { beaker }\end{array}$ & ND & 0 & 0 & ND & 0 & $0 \%$ & ND & 0 & 0 & ND & 0 & 0 \\
\hline
\end{tabular}

Deep Scan Time=DST, Total No of Files extracted out of 1GB= FS, Recovery status =\% R, Not Detected=ND

Table 3.3 Heating Method

Method 3.3: Hot Oven Method

\begin{tabular}{|c|c|c|c|c|c|c|c|c|c|c|c|c|c|}
\hline \multirow[t]{2}{*}{$\begin{array}{c}\text { Sampl } \\
\text { e No }\end{array}$} & \multirow{2}{*}{$\begin{array}{c}\text { Conditio } \\
\mathbf{n}\end{array}$} & \multicolumn{3}{|c|}{$\begin{array}{c}\text { Software Tool No1 } \\
\text { Recuva } \\
\text { Freeware }\end{array}$} & \multicolumn{3}{|c|}{$\begin{array}{c}\text { Software Tool No } 2 \\
\text { Photorec 7.0 Data } \\
\text { Recovery } \\
\text { Freeware } \\
\end{array}$} & \multicolumn{3}{|c|}{$\begin{array}{l}\text { Software Tool No } 3 \\
\text { 7-Data Recovery }\end{array}$} & \multicolumn{3}{|c|}{$\begin{array}{c}\text { Software Tool No } 4 \\
\text { Stellar Phoenix } \\
\text { Licensed }\end{array}$} \\
\hline & & $\begin{array}{c}\text { DS } \\
\text { T }\end{array}$ & $\begin{array}{l}\text { FSE } \\
(269 \\
\text { (269 }\end{array}$ & $\mathbf{R}$ & $\begin{array}{c}\text { DS } \\
\text { T }\end{array}$ & $\begin{array}{l}\text { FSE } \\
(269 \\
\text { ) }\end{array}$ & $\mathbf{R}$ & DST & $\begin{array}{c}\text { FSE } \\
(269 \\
)\end{array}$ & $\mathbf{R}$ & $\begin{array}{c}\text { DS } \\
\text { T }\end{array}$ & $\begin{array}{l}\text { FSE } \\
(269 \\
)\end{array}$ & $\mathbf{R}$ \\
\hline $\begin{array}{l}\text { Sampl } \\
\text { e } 1\end{array}$ & 80 & $\begin{array}{l}3.57 \\
\text { HR } \\
\text { S }\end{array}$ & 230 & $942 \mathrm{MB}$ & $\begin{array}{l}1.36 \\
\mathrm{HR} \\
\mathrm{S}\end{array}$ & 275 & $\begin{array}{l}989 \mathrm{M} \\
\text { B }\end{array}$ & $\begin{array}{l}1.35 \\
\text { HRS }\end{array}$ & 506 & $\begin{array}{l}1.66 \mathrm{G} \\
\text { B }\end{array}$ & $\begin{array}{l}1.6 \\
\text { HR } \\
S\end{array}$ & 270 & $\begin{array}{l}1.0 \mathrm{G} \\
\mathrm{B}\end{array}$ \\
\hline $\begin{array}{l}\text { Sampl } \\
\text { e } 2\end{array}$ & 110 & $\begin{array}{l}3.56 \\
\text { HR } \\
\text { S }\end{array}$ & 29 & $\begin{array}{l}2.58 \mathrm{G} \\
\mathrm{B}\end{array}$ & $\begin{array}{l}2 \\
\mathrm{HR} \\
\mathrm{S}\end{array}$ & 275 & $\begin{array}{l}989 \mathrm{M} \\
\text { B }\end{array}$ & $\begin{array}{l}1.56 \\
\text { HRS }\end{array}$ & 506 & $\begin{array}{l}1.66 \mathrm{G} \\
\text { B }\end{array}$ & $\begin{array}{l}1.6 \\
\text { HR } \\
\text { S }\end{array}$ & 270 & $\begin{array}{l}1.0 \mathrm{G} \\
\mathrm{B}\end{array}$ \\
\hline $\begin{array}{l}\text { Sampl } \\
\text { e } 3\end{array}$ & 160 & $\begin{array}{l}3.45 \\
\text { HR } \\
\text { S }\end{array}$ & 28 & $\begin{array}{l}2.80 \mathrm{G} \\
\mathrm{B}\end{array}$ & $\begin{array}{l}1.56 \\
\mathrm{HR} \\
\mathrm{S}\end{array}$ & 275 & $\begin{array}{l}989 \mathrm{M} \\
\text { B }\end{array}$ & $\begin{array}{l}1.56 \\
\text { HRS }\end{array}$ & 506 & $\begin{array}{l}1.66 \mathrm{G} \\
\mathrm{B}\end{array}$ & $\begin{array}{l}1.6 \\
\text { HR } \\
\text { S }\end{array}$ & 270 & $\begin{array}{l}1.0 \mathrm{G} \\
\mathrm{B}\end{array}$ \\
\hline $\begin{array}{l}\text { Sampl } \\
\text { e } 4\end{array}$ & $\begin{array}{l}\text { Heated } 5 \\
\text { sec }\end{array}$ & ND & 0 & 0 & ND & 0 & 0 & $\begin{array}{l}\text { SD- } \\
\text { NDS } \\
\mathrm{T}\end{array}$ & 0 & 0 & ND & 0 & 0 \\
\hline $\begin{array}{l}\text { Sampl } \\
\text { e } 5\end{array}$ & $\begin{array}{l}\text { Heated } \\
60 \mathrm{sec}\end{array}$ & ND & 0 & 0 & ND & 0 & 0 & ND & 0 & 0 & ND & 0 & 0 \\
\hline
\end{tabular}

Deep Scan Time=DST, Total No of Files extracted out of $1 \mathrm{~GB}=\mathrm{FS}$, Recovery status $=\mathrm{R}$, Not Detected=ND,

Successfully detected but No DST processed=SD-NDST

Table 3.4 Freezing Method

Method 3.4: Freezing Method

\begin{tabular}{|c|c|c|c|c|c|c|c|c|c|c|c|c|c|}
\hline \multirow[t]{2}{*}{$\begin{array}{l}\text { Sampl } \\
\text { e No }\end{array}$} & \multirow{2}{*}{$\begin{array}{l}\text { Conditi } \\
\text { on }\end{array}$} & \multicolumn{3}{|c|}{$\begin{array}{c}\text { Software Tool No1 } \\
\text { Recuva } \\
\text { Freeware }\end{array}$} & \multicolumn{3}{|c|}{$\begin{array}{c}\text { Software Tool No } 2 \\
\text { Photorec 7.0 Data } \\
\text { Recovery } \\
\text { Freeware }\end{array}$} & \multicolumn{3}{|c|}{$\begin{array}{c}\text { Software Tool No } 3 \\
\text { 7-Data Recovery }\end{array}$} & \multicolumn{3}{|c|}{$\begin{array}{c}\text { Software Tool No } 4 \\
\text { Stellar Phoenix } \\
\text { Licensed }\end{array}$} \\
\hline & & $\begin{array}{c}\text { DS } \\
\mathbf{T}\end{array}$ & $\begin{array}{l}\text { FSE } \\
(269 \\
)\end{array}$ & $\mathbf{R}$ & DST & $\begin{array}{l}\text { FSE } \\
(269 \\
)\end{array}$ & $\mathbf{R}$ & DST & $\begin{array}{l}\text { FSE } \\
(269 \\
)\end{array}$ & $\mathbf{R}$ & $\begin{array}{c}\text { DS } \\
\text { T }\end{array}$ & $\begin{array}{l}\text { FSE } \\
(269 \\
)\end{array}$ & $\mathbf{R}$ \\
\hline $\begin{array}{l}\text { Sampl } \\
\text { e } 1\end{array}$ & $1 \mathrm{HR}$ & $\begin{array}{c}2.1 \\
5 \\
\mathrm{HR} \\
\mathrm{S}\end{array}$ & 295 & 30.6GB & $\begin{array}{c}3.56 \mathrm{HR} \\
\mathrm{S}\end{array}$ & 274 & $\begin{array}{c}989 \mathrm{M} \\
\mathrm{B}\end{array}$ & $\begin{array}{l}2.15 \\
\text { HRS }\end{array}$ & 506 & $\begin{array}{c}1.66 \mathrm{G} \\
\mathrm{B}\end{array}$ & $\begin{array}{c}2.5 \\
6\end{array}$ & 270 & $\begin{array}{c}1.66 \mathrm{G} \\
\mathrm{B}\end{array}$ \\
\hline $\begin{array}{l}\text { Sampl } \\
\text { e } 2\end{array}$ & 24 HRS & $\begin{array}{c}2.1 \\
5 \\
\mathrm{HR} \\
\mathrm{S}\end{array}$ & 4 & 2.49GB & $\begin{array}{l}1.57 \\
\text { HRS }\end{array}$ & 274 & $\begin{array}{c}989 \mathrm{M} \\
\mathrm{B}\end{array}$ & $\begin{array}{l}1.56 \\
\text { HRS }\end{array}$ & 506 & $\begin{array}{c}1.66 \mathrm{G} \\
\mathrm{B}\end{array}$ & $\begin{array}{c}3.4 \\
5\end{array}$ & 270 & $\begin{array}{c}1.66 \mathrm{G} \\
\mathrm{B}\end{array}$ \\
\hline $\begin{array}{l}\text { Sampl } \\
\text { e } 3\end{array}$ & 48 HRS & $\begin{array}{c}2.1 \\
5 \\
\mathrm{HR} \\
\mathrm{S} \\
\end{array}$ & 200 & $\begin{array}{c}74.349 .95 \\
\text { GB }\end{array}$ & $\begin{array}{l}2.56 \\
\text { HRS }\end{array}$ & 274 & $\begin{array}{c}989 \mathrm{M} \\
\mathrm{B}\end{array}$ & $\begin{array}{l}1.59 \\
\text { HRS }\end{array}$ & 506 & $\begin{array}{c}1.66 \mathrm{G} \\
\mathrm{B}\end{array}$ & $\begin{array}{c}3.1 \\
0\end{array}$ & 270 & $\begin{array}{c}1.66 \mathrm{G} \\
\mathrm{B}\end{array}$ \\
\hline
\end{tabular}




\begin{tabular}{|c|c|c|c|c|c|c|c|c|c|c|c|c|c|}
\hline $\begin{array}{c}\text { Sampl } \\
\text { e } 4\end{array}$ & 72 HRS & $\begin{array}{c}2.1 \\
5 \\
\mathrm{HR} \\
\mathrm{S}\end{array}$ & 59 & $8.26 \mathrm{~GB}$ & $\begin{array}{l}3.00 \\
\text { HRS }\end{array}$ & 274 & $\begin{array}{c}921 \mathrm{M} \\
\mathrm{B}\end{array}$ & $\begin{array}{c}2.19 \mathrm{HR} \\
\mathrm{S}\end{array}$ & 506 & $\begin{array}{c}1.66 \mathrm{G} \\
\text { B }\end{array}$ & $\begin{array}{c}3.3 \\
4\end{array}$ & 270 & $\begin{array}{c}1.66 \mathrm{G} \\
\text { B }\end{array}$ \\
\hline $\begin{array}{c}\text { Sampl } \\
\text { e } 5\end{array}$ & 96 HRS & $\begin{array}{c}2.1 \\
5 \\
\text { HR } \\
S\end{array}$ & 258 & 13.9GB & $\begin{array}{l}1.56 \\
\text { HRS }\end{array}$ & 274 & $\begin{array}{c}989 \mathrm{M} \\
\mathrm{B}\end{array}$ & $\begin{array}{l}2.10 \\
\text { HRS }\end{array}$ & 506 & $\begin{array}{c}1.66 \mathrm{G} \\
\mathrm{B}\end{array}$ & $\begin{array}{c}3.2 \\
3\end{array}$ & 270 & $\begin{array}{c}1.66 \mathrm{G} \\
\mathrm{B}\end{array}$ \\
\hline
\end{tabular}

Deep Scan Time=DST, Total No of Files extracted out of 1GB= FS, Recovery status $=\mathrm{R}$

Table 3.5 Scratching Method

Method 3.5: Scratch Method

\begin{tabular}{|c|c|c|c|c|c|c|c|c|c|c|c|c|c|}
\hline \multirow[t]{2}{*}{$\begin{array}{l}\text { Sampl } \\
\text { e No }\end{array}$} & \multirow{2}{*}{$\begin{array}{c}\text { Conditio } \\
\mathbf{n}\end{array}$} & \multicolumn{3}{|c|}{$\begin{array}{c}\text { Software Tool No1 } \\
\text { Recuva } \\
\text { Freeware }\end{array}$} & \multicolumn{3}{|c|}{$\begin{array}{c}\text { Software Tool No } 2 \\
\text { Photorec 7.0 Data } \\
\text { Recovery } \\
\text { Freeware } \\
\end{array}$} & \multicolumn{3}{|c|}{$\begin{array}{l}\text { Software Tool No } 3 \\
\text { 7-Data Recovery }\end{array}$} & \multicolumn{3}{|c|}{$\begin{array}{c}\text { Software Tool No } 4 \\
\text { Stellar Phoenix } \\
\text { Licensed }\end{array}$} \\
\hline & & DST & $\begin{array}{l}\text { FSE } \\
(269 \\
) \\
\end{array}$ & $\% R$ & $\begin{array}{c}\text { DS } \\
\text { T }\end{array}$ & $\begin{array}{c}\text { FSE } \\
(269 \\
) \\
\end{array}$ & $\% \mathrm{R}$ & $\begin{array}{c}\text { DS } \\
\mathbf{T}\end{array}$ & $\begin{array}{c}\text { FSE } \\
(269 \\
)\end{array}$ & $\% \mathrm{R}$ & $\begin{array}{c}\text { DS } \\
\mathbf{T}\end{array}$ & $\begin{array}{c}\text { FSE } \\
(269 \\
)\end{array}$ & $\% R$ \\
\hline $\begin{array}{l}\text { Sampl } \\
\text { e } 1\end{array}$ & 100 & $\begin{array}{c}2.45 \mathrm{HR} \\
\mathrm{S}\end{array}$ & 295 & $\begin{array}{l}30.6 \mathrm{G} \\
\mathrm{B}\end{array}$ & $\begin{array}{c}2.14 \\
\mathrm{HR} \\
\mathrm{S}\end{array}$ & 274 & $\begin{array}{l}989 \mathrm{M} \\
\text { B }\end{array}$ & $\begin{array}{c}2.45 \\
\text { HR } \\
\text { S }\end{array}$ & 506 & $\begin{array}{l}1.66 \mathrm{G} \\
\mathrm{B}\end{array}$ & $\begin{array}{c}1.56 \\
\text { HR } \\
\text { S }\end{array}$ & 270 & $\begin{array}{l}1.0 \mathrm{G} \\
\mathrm{B}\end{array}$ \\
\hline $\begin{array}{c}\text { Sampl } \\
\text { e } 2\end{array}$ & 150 & $\begin{array}{l}3.23 \\
\text { HRS }\end{array}$ & 295 & $\begin{array}{l}30.6 \mathrm{G} \\
\mathrm{B}\end{array}$ & $\begin{array}{c}2.56 \\
\text { HR } \\
\text { S }\end{array}$ & 274 & $\begin{array}{l}989 \mathrm{M} \\
\text { B }\end{array}$ & $\begin{array}{c}3.56 \\
\text { HR } \\
\text { S }\end{array}$ & 506 & $\begin{array}{l}1.66 \mathrm{G} \\
\mathrm{B}\end{array}$ & $\begin{array}{c}2.34 \\
\text { HR } \\
\text { S }\end{array}$ & 270 & $\begin{array}{l}1.0 \mathrm{G} \\
\mathrm{B}\end{array}$ \\
\hline $\begin{array}{l}\text { Sampl } \\
\text { e } 3\end{array}$ & 200 & $\begin{array}{l}3.12 \\
\text { HRS }\end{array}$ & 295 & $\begin{array}{l}30.6 \mathrm{G} \\
\mathrm{B}\end{array}$ & $\begin{array}{c}3.00 \\
\mathrm{HR} \\
\mathrm{S}\end{array}$ & 274 & $\begin{array}{l}989 \mathrm{M} \\
\text { B }\end{array}$ & $\begin{array}{c}3.12 \\
\text { HR } \\
\text { S }\end{array}$ & 506 & $\begin{array}{l}\text { 1.66G } \\
\text { B }\end{array}$ & $\begin{array}{c}3.45 \\
\text { HR } \\
\text { S }\end{array}$ & 270 & $\begin{array}{l}1.0 \mathrm{G} \\
\mathrm{B}\end{array}$ \\
\hline $\begin{array}{l}\text { Sampl } \\
\text { e } 4\end{array}$ & 250 & $\begin{array}{l}3.56 \\
\text { HRS }\end{array}$ & 295 & $\begin{array}{l}30.6 \mathrm{G} \\
\mathrm{B}\end{array}$ & $\begin{array}{c}2.34 \\
\mathrm{HR} \\
\mathrm{S} \\
\end{array}$ & 274 & $\begin{array}{l}989 \mathrm{M} \\
\text { B }\end{array}$ & $\begin{array}{c}3.23 \\
\mathrm{HR} \\
\mathrm{S}\end{array}$ & 506 & $\begin{array}{l}1.66 \mathrm{G} \\
\mathrm{B}\end{array}$ & $\begin{array}{c}4.00 \\
\text { HR } \\
\text { S }\end{array}$ & 270 & $\begin{array}{l}1.0 \mathrm{G} \\
\mathrm{B}\end{array}$ \\
\hline $\begin{array}{l}\text { Sampl } \\
\text { e } 5 \\
\end{array}$ & 500 & ND & 0 & 0 & ND & 0 & 0 & ND & 0 & 0 & ND & 0 & 0 \\
\hline
\end{tabular}

Deep Scan Time=DST, Total No of Files extracted out of $1 \mathrm{~GB}=\mathrm{FS}$, Recovery status $=\mathrm{R}$, Not Detected=N

\section{CONCLUSION}

In Case of MICRO SD CARD memory card,

- $\quad$ Due To Damaged MICRO SD CARD

Hypothesis proved to be wrong as evidences from experimental results data can be recovered from damaged MICRO SD CARD memory cards can be achieved.

- Comparisms between software's using various parameters were observed.

- Stellar phoenix as it was licensed software gives $100 \%$ result with little noise insertion. While other three software maximum noise is inserted like in form of (Wed,To,TED,OY,OUT,CESa,ITH,ION,ING,ILE,IA,I< ,HFA, $\mathrm{H}<(\mathrm{E}, \mathrm{H}<$,Fix,File,EAD,ATE,ATA,AND,AFL, \%f ",s",SD ...etc)

- In freeware software Photorec 7.0 Data Recovery, 7Data Recovery and then Recuva give good results respectively.

- Noise was inserted because of damage done to devices, because of which maximum data space is required to store the data. Redundant data was seen present mainly in Recuva.

- It is concluded that data can be recovered is possible in either Full recovery, partially recovery and in very rare cases at extreme conditions data is completely loss hence recovery is just impossible.

\section{REFERENCES}

1. Alpna, Dr. Sona Malhotra ," Cyber Crime-Its Types, Analysis and Prevention Techniques" International Journal of Advanced Research in Computer Science and Software Engineering Research Volume 6, Issue 5, May 2016 ISSN: 2277 128X ,page no 145. Paper Available online at: www.ijarcsse.com

2. Thomas A. Johnson chapter No 1 "Forensic Computer Crime investigation" CRC Taylor \& Francis.

3. https://www.statista.com/statistics/553556/worldwide-flash-memorymarket-size/

4. Constantine., Photopoulos, (2008). Managing catastrophic loss of sensitive data : a guide for IT and security professionals. Rockland, Mass.: Syngress. ISBN 9781597492393. OCLC 228148168.

5. UFS Explorer and data recovery and access software ,'Knowledge Base"

https://www.ufsexplorer.com/articles/what-is-data-recovery.php

6. Oliver Powell ,"What is Data Recovery and How It is Helpful for You?"Updated on January 14, 2019, https://www.stellarinfo.com/blog/know-about-data-recovery/

7. SanDisk Secure Digital (SD) Card Product Manual, Rev. 1.9 (C) 2003 SANDISK CORPORATION "Introduction to the SD Card" chapter 1. Page no 1-2 www.sandisk.com

8. Sneha Pandhare, Dr.Shobha Bawiskar," Recovery Of Data From Damaged Disks".(Online-Oral Presentation), International Conference on "Innovations in Engineering, Technology and Sciences"- (ICIETS2018) with catlog "CFP18Q63-PRJ:978-1-53867321-8" held on September 21-22 ,2018, NIE Institute of Technology, Mysore, Karnataka, (Bangalore)India, will be published in IEEE Xplore Digital Library

9. Recuva software https://recuva.en.softonic.com/downloadStellar Phoneix 
Determining the Scope of Recovery from Physically Damaged Micro SD Card

10. 7 Data Recovery Software

https://7datarecovery.com/\#forwardPhotrec data recovery

11. Photorec $\quad \mathbf{7 . 0} \quad$ Data $\quad$ Recovery

https://downloads.tomsguide.com/PhotoRec,0301-32874.html

12. Stellar Phoneix : https://www.stellari nfo.com/ 